SLAC-PUB-8341

January 2000

\title{
Rapidity Gaps From Color String Topologies
}

\author{
G. Ingelman et al.
}

Contributed to 7th International Workshop on Deep Inelastic Scattering and QCD (DIS 99), 4/19/1999—4/23/1999, Zeuthen, Germany 


\title{
Rapidity Gaps from Colour String Topologies*
}

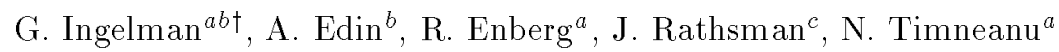 \\ a High Energy Physics, Uppsala University, Box 535, S-751 21 Uppsala, Sweden \\ ${ }^{b}$ DESY, Notkestrasse 85, D-22603 Hamburg, Germany \\ ${ }^{c}$ SLAC, Stanford, California 94309, USA
}

Diffractive deep inelastic scattering at HERA and diffractive $W$ and jet production at the Tevatron are well described by soft colour exchange models. Their essence is the variation of colour string-field topologies giving both gap and no-gap events, with a smooth transition and thereby a unified description of all final states.

The hard scale in diffractive hard scattering [1, 5 , has provided the possibility to analyse rapidity gap events based on underlying parton processes calculable in perturbation theory. Although this has been quite successful, perturbative QCD (PQCD) cannot give the complete solution since the rapidity gap connects to the soft part of the event where non-perturbative effects on a long space-time scale are important.

In order to understand these non-perturbative effects and provide a unified description of all final states, we have developed models for the soft dynamics. These models are added to Monte Carlo

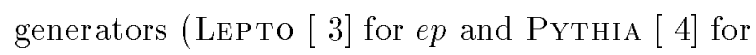
$p \bar{p}$ ), such that an experimental approach can be taken to classify events depending on the characteristics of the final state: e.g. gaps or no-gaps, leading protons or neutrons etc.

The basic assumption of the models is that variations in the topology of the confining colour force fields (strings) lead to different hadronic final states after hadronisation (Fig. 1 i 1 i ). The PQCD interaction gives a set of partons with a specific colour order. However, this order may change due to soft, non-perturbative interactions.

In the soft colour interaction (SCI) model [ [ $\left[\begin{array}{l}1-5 \\ 0-1\end{array}\right]$ it is assumed that colour-anticolour, corresponding to non-perturbative gluons, can be exchanged between partons and remnants emerging from a hard scattering. This can be viewed as the partons interacting softly with the colour medium of

\footnotetext{
*Contribution to the DIS 99 workshop proceedings

$\dagger$ ingelman@desy.de
}

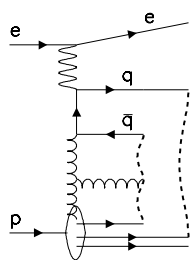

(a)

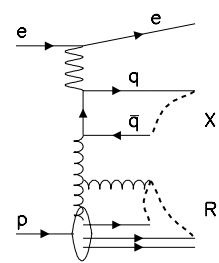

(b)

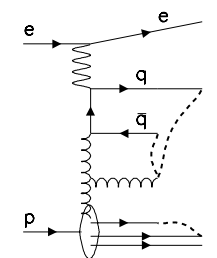

(c)
Figure 1. Gluon-induced DIS event with examples of colour string connection (dashed lines) of partons in (a) conventional Lund model based on the colour order in PQCD, and $(b, c)$ after colour rearrangement through SCI or GAL mechanisms.

the proton as they propagate through it, which should be a natural part of the process in which 'bare' perturbative partons are 'dressed' into nonperturbative ones and the confining colour flux tube between them is formed. The hard parton level interactions are given by standard perturbative matrix elements and parton showers, which are not altered by softer non-perturbative effects. The unknown probability to exchange a soft gluon between parton pairs is given by a phenomenological parameter $R$, which is the only free parameter of the model. With $R=0.5$ one obtains the correct rate of rapidity gap events observed at HERA and a quite decent description of the measured diffractive structure function $\left[\begin{array}{l}\overline{6} \\ 1\end{array}\right.$ (Fig. $\overline{2}$.

Leading neutrons are also obtained in agree- 


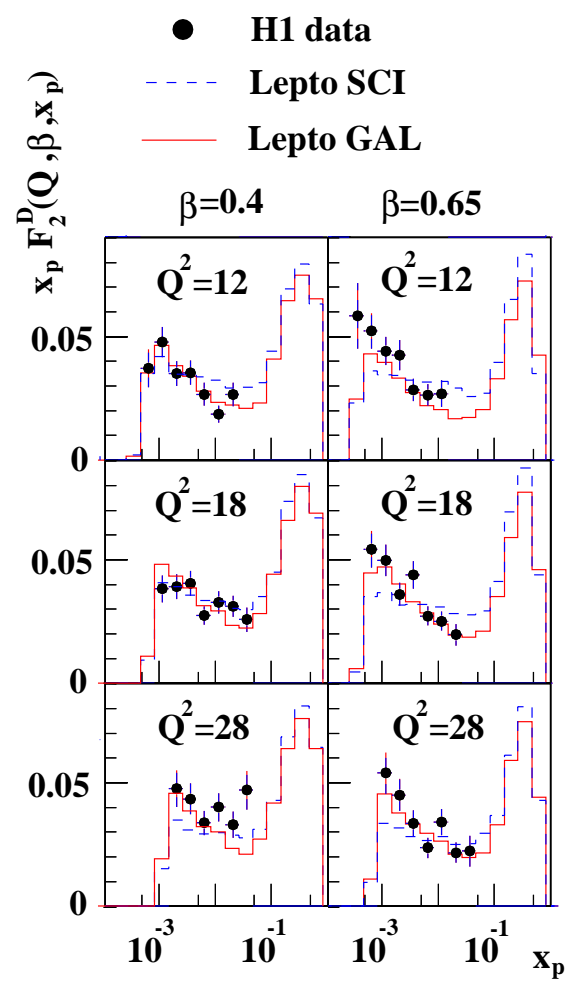

Figure 2. The diffractive structure function [ $\left[\begin{array}{l}8 \\ 8\end{array}\right]$.

ment with experimental measurements [ $\overline{7} \bar{i}$. In the Regge approach pomeron exchange would be used for diffraction, pion exchange added to get leading neutrons and still other exchanges should be added for completeness. The SCI model provides a simpler description.

Applying the same SCI model to hard $p \bar{p}$ collisions one obtains production of $W$ and di-jets in association with rapidity gaps (Fig. ing the $R$-value obtained from gaps at HERA, the observed rates of diffractive $W$ and diffractive di-jet production at the Tevatron are reproduced (Fig. which, when tuned to HERA gap events, gives a factor $\sim 6$ too large rate at the Tevatron [ $\left[\begin{array}{c}127 \\ 27\end{array}\right]$

SCI does not only lead to rapidity gaps, but also to other striking effects. It reproduces [ the observed rate of high- $p_{\perp}$ charmonium and bottomonium at the Tevatron, which are factors of 10 larger than predictions based on conventional PQCD. This is accomplished by the change
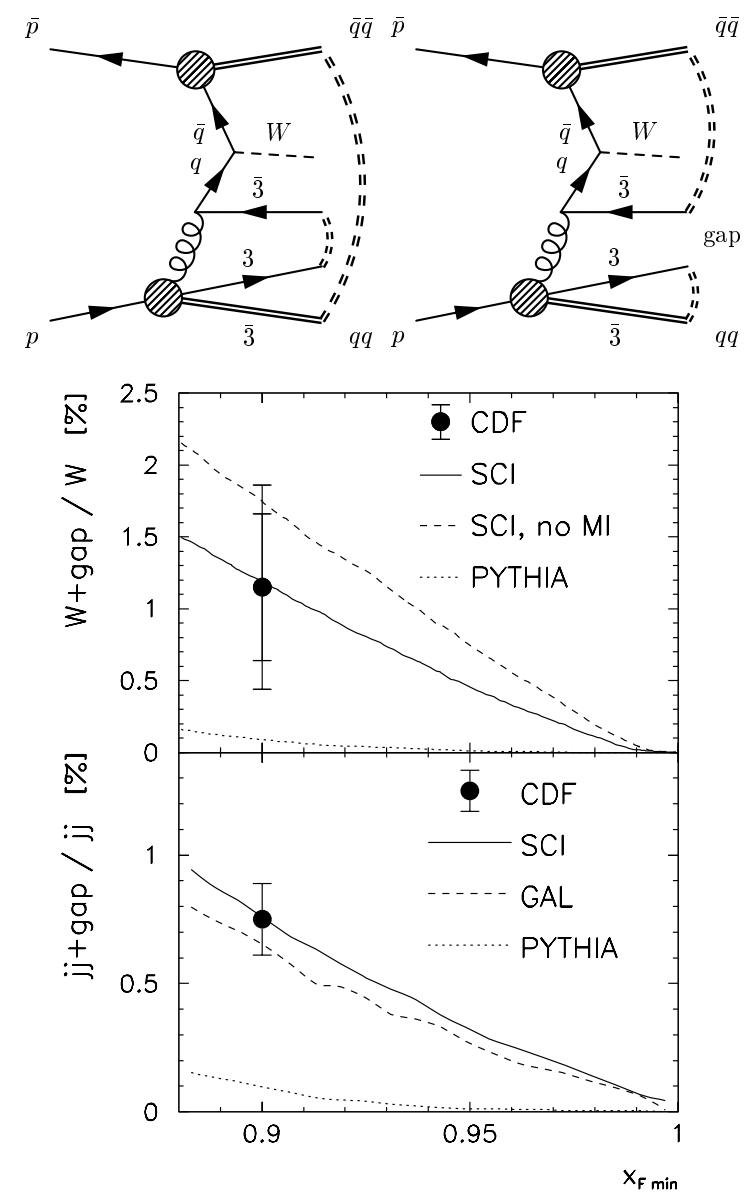

Figure 3. $W$ production in $p \bar{p}$ with string topology before and after colour rearrangement resulting in a gap. Relative rate of $W$ and di-jet events with a rapidity gap corresponding to diffraction with a leading proton with minimum $x_{F}=0.9$ in Tevatron data [ 110.0 and in the SCI and GAL models. Sensitivity to multiparton interactions (MI) and results without SCI or GAL is shown [ 9.1.

of the colour charge of a $Q \bar{Q}$ pair (e.g. from a gluon) from octet to singlet. A quarkonium state can then be formed using a simple model for the division of the cross-section below the threshold for open heavy flavour production onto different quarkonium states.

An alternative to SCI is the newly developed

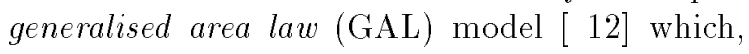


based on a generalisation of the area law suppression $e^{-b A}$ with $A$ the area swept out by the string in energy-momentum space, gives modified colour string topologies through string reinteractions. The probability $P=R_{0}[1-\exp (-b \Delta A)]$ for two strings pieces to interact depends on the area difference $\Delta A$ which is gained by the string rearrangement. This favours making 'shorter' strings, e.g. with gaps, whereas making 'longer', 'zig-zag' shaped strings is suppressed. The fixed probability $R$ in SCI is thus replaced by a dynamical one, where the parameter $R_{0}=0.1$ is chosen to reproduce the HERA gap event rate in a simultaneous fit to data from $e^{+} e^{-}$annihilation at the $Z^{0}$-peak. The resulting diffractive structure function compares very well with HERA data (Fig. 20 ). The GAL model also improves the description of non-diffractive HERA data [

The GAL model can also be applied to $p \bar{p}$ to obtain diffractive $W$ and di-jet production through string rearrangements like in Fig. rates are reproduced quite well (Fig. $\overline{p_{1}^{\prime}}$ ). However, the treatment of the 'underlying event', which is a notorious problem in hadron-hadron scattering, introduces a larger uncertainty than for the SCI model [ $19 \overline{9}$.

The Tevatron data on gaps between two high$p_{\perp}$ jets are harder to understand. SCI does give such events, but at a too low rate. The GAL model can give the observed rate, but again with an uncertainty due to the treatment of the underlying event. The measured colour-singlet fraction in D0 [ 193inds to increase with increasing jet separation, whereas CDF data [ [1 $\left.1 \overline{4}_{1}^{1}\right]$ shows no significant such effect. However, the required gap size is fixed to $-1<\eta<1$. Our Monte Carlo study shows an increase with jet separation with this fixed gap size, but a decrease when the gap size follows the jet separation (Fig. $\bar{i}$ i. . The proper diffractive signature should rather be no suppression with increasing gap size. Thus, the exact gap definition is very important for the interpretation and this issue should therefore be examined further experimentally.

In conclusion, our models for non-perturbative QCD dynamics in terms of varying colour string topologies give a satisfactory explanation of several phenomena, both diffractive and non-

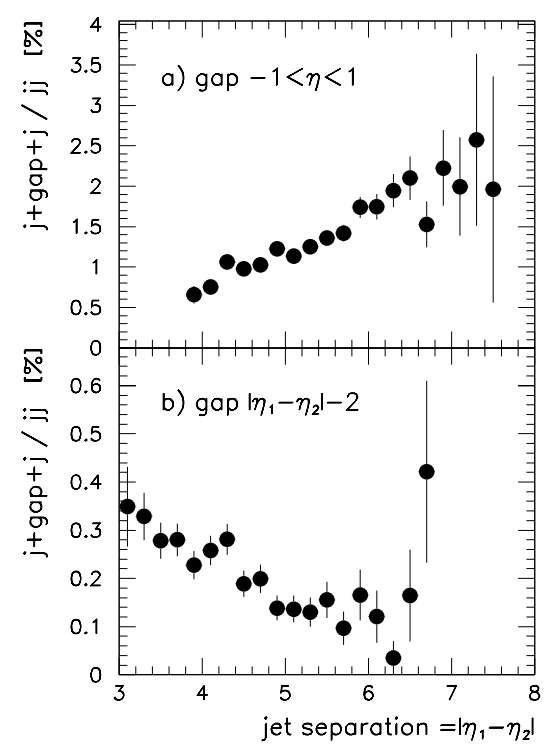

Figure 4. Relative rates of gaps between jets from GAL model simulations of Tevatron $p \bar{p}$ with the gap size (a) fixed and (b) increasing with the jet separation $[\bar{q}]$.

diffractive, thus providing a unified description of many different hadronic final states.

\section{REFERENCES}

1. G. Ingelman, P.E. Schlein, Phys. Lett. B152 (1985) 256

2. For a review, see G. Ingelman, DESY 99-009

3. G. Ingelman, A. Edin, J. Rathsman, Comput. Phys. Commun. 101 (1997) 108

4. T. Sjöstrand, Comput. Phys. Commun. 82 (1994) 74

5. A. Edin, G. Ingelman, J. Rathsman, Phys. Lett. B366 (1996) 371; Z.Phys. C75 (1997) 57

6. C. Adloff et al. (H1), Z. Phys. C76 (1997) 613

7. C. Adloff et al., Eur. Phys. J. C6 (1999) 587

8. A. Edin, G. Ingelman, J. Rathsman, in preparation

9. R. Enberg, G. Ingelman, N. Timneanu, in preparation

10. F. Abe et al.(CDF), Phys.Rev.Lett. 78 (1997) 2698; Phys. Rev. Lett. 79 (1997) 2636

11. A. Edin, G. Ingelman, J. Rathsman, Phys. Rev. D56 (1997) 7317

12. J. Rathsman, Phys. Lett. B452 (1999) 364 
13. B. Abbott et al., Phys. Lett. B440 (1998) 189

14. F. Abe et al., Phys. Rev. Lett. 80 (1998) 1156; ibid. 81 (1998) 5278 